\title{
Existence of traveling wave solutions in m-dimensional delayed lattice dynamical systems with competitive quasimonotone and global interaction
}

\author{
Kai Zhou \\ Department of Mathematics, Shanghai Normal University, Shanghai 200234, P. R. China. \\ School of Mathematics and Computer, Chizhou University, Chizhou 247000, P. R. China.
}

Communicated by K. Q. Lan

\begin{abstract}
This paper deals with the existence of traveling wave solutions for $m$-dimensional delayed lattice dynamical systems with competitive quasimonotone and global interaction. By using Schauder's fixed point theorem and a cross-iteration scheme, we reduce the existence of traveling wave solutions to the existence of a pair of upper and lower solutions. The general results obtained will be applied to m-dimensional delayed lattice dynamical systems with Lotka-Volterra type competitive reaction terms and global interaction. (C)2017 All rights reserved.
\end{abstract}

Keywords: Traveling wave solutions, lattice differential systems, delay, upper and lower solutions, Schauder's fixed point theorem.

2010 MSC: 34A33, 34K31, 92D25.

\section{Introduction}

Lattice differential systems are infinite systems of ordinary differential equations indexed by points in a lattice, such as the D-dimensional integer lattice $\mathbb{Z}^{\mathrm{D}}$ which involve some aspect of the spatial structure of the lattice. Such systems arise from practical backgrounds, such as modeling population growth over patchy environments $[8,16,20]$ and modeling the phase transitions $[1,2]$. On the other hand, they are also the natural results of discretization of spatial variable for the continuous models such as partial differential equations $[7,9,18]$. We refer to the surveys of lattice dynamical systems by Chow [6]. Particularly, many researchers have paid attention to the traveling wave solutions of lattice dynamical systems due to its significant sense in mathematical theory and practical fields. More precisely, they can determine the asymptotical behavior of the corresponding initial value problem for the lattice dynamical systems, and also describe many important phenomena in population dynamics, physical science and other practical areas.

In the past years, traveling wave solutions for lattice differential equations with or without time delays have been widely studied by many authors. For the single lattice differential equation, Bell and Cosner

Email address: zk1984@163.com (Kai Zhou)

doi:10.22436/jnsa.010.07.23 
[3] used

$$
\frac{d u_{n}(t)}{d t}=D\left[u_{n+1}(t)-2 u_{n}(t)+u_{n-1}(t)\right]+f\left(u_{n}(t)\right), \quad n \in \mathbb{Z}, \quad t>0,
$$

to model myelinated axons in nerve systems. They studied the long time behavior of solutions to (1.1) for some nonlinear function $f$. When the nonlinear term $f$ is a monostable/bistable type, there are extensive results about the traveling wave solutions for equation (1.1), some of which have revealed some essential differences between a discrete model and its corresponding continuous one. For details, see for example, $[4,5,23,24]$. Taking into account time delay in population dynamics, $\mathrm{Wu}$ and $\mathrm{Zou}$ [21] considered the delayed lattice differential equations and studied the existence of traveling wave solutions.

As mentioned in Weinberger et al. [19], the most interesting population model should involve the interactions of different species, and there are also some concrete system of lattice differential equations which are derived in population dynamics. For example, Huang and Lu [10] and Huang et al. [11] considered the following delayed lattice dynamical systems

$$
\left\{\begin{array}{l}
\frac{d u_{n}(t)}{d t}=\sum_{j=1}^{m} a_{j}\left[g\left(u_{n+j}(t)\right)-2 g\left(u_{n}(t)\right)+g\left(u_{n-j}(t)\right)\right]+f_{1}\left(u_{n}(t-\tau), v_{n}(t-\tau)\right), \\
\frac{d v_{n}(t)}{d t}=\sum_{j=1}^{m} a_{j}\left[g\left(v_{n+j}(t)\right)-2 g\left(v_{n}(t)\right)+g\left(v_{n-j}(t)\right)\right]+f_{2}\left(u_{n}(t-\tau), v_{n}(t-\tau)\right)
\end{array}\right.
$$

where $n \in \mathbb{Z}, m \geqslant 1$ is an integer, $a_{j}, b_{j}>0, f_{i}: C\left([-\tau, 0], \mathbb{R}^{2}\right) \rightarrow \mathbb{R}$ and $g: \mathbb{R} \rightarrow \mathbb{R}$ are continuous functions. By using the method of monotone iteration and upper-lower solution, they established the existence of traveling wave solutions for system (1.2) connecting the trivial equilibrium and the positive one, if the reaction terms satisfy the so-called (exponential) quasimonotone condition or the partial (exponential) quasimonotone condition. Later, Lin and Li [12] considered the delayed system of two lattice ODEs

$$
\left\{\begin{array}{l}
\frac{d u_{n}(t)}{d t}=g_{1}\left(u_{n+1}(t)\right)+g_{1}\left(u_{n-1}(t)\right)-2 g_{1}\left(u_{n}(t)\right)+f_{1}\left(u_{n t}, v_{n t}\right), \\
\frac{d v_{n}(t)}{d t}=g_{2}\left(v_{n+1}(t)\right)+g_{2}\left(v_{n-1}(t)\right)-2 g_{2}\left(v_{n}(t)\right)+f_{2}\left(u_{n t}, v_{n t}\right),
\end{array}\right.
$$

where $f_{i}, i=1,2$ satisfy the (exponential) competitive quasimonotone condition, and obtained the existence of traveling wave solutions for system (1.3).

Besides the lattice dynamical systems mentioned above, there are also some literatures consider the lattice differential equations with global interaction. For example, Ma et al. [17] derived a discrete model for a single species in one-dimensional patchy environment with infinite number of patches connected non-locally by diffusion, which takes the form

$$
\frac{d u_{n}(t)}{d t}=\sum_{i \in \mathbb{Z}_{0}} J(i)\left[u_{n+i}(t)-u_{n}(t)\right]-d u_{n}(t)+\sum_{i \in \mathbb{Z}} K(i) b\left(u_{n-i}(t-\tau)\right), \quad n \in \mathbb{Z} .
$$

Recently, Lin et al. [14] considered the traveling wavefronts of the following general system of lattice differential equations

$$
\begin{aligned}
\frac{d u_{n}^{i}(t)}{d t}= & \sum_{k \in \mathbb{Z}_{0}} J_{i}(k)\left[u_{n+k}^{i}(t)-u_{n}^{i}(t)\right] \\
& +f_{i}\left(\sum_{l \in \mathbb{Z}} J_{i 1}(l) u_{n+l}^{1}\left(t-\tau_{i 1}\right), \cdots, \sum_{l \in \mathbb{Z}} J_{i m}(l) u_{n+l}^{m}\left(t-\tau_{i m}\right)\right), \quad n \in \mathbb{Z},
\end{aligned}
$$

where $u_{n}=\left(u_{n}^{1}, u_{n}^{2}, \cdots, u_{n}^{m}\right) \in \mathbb{R}^{m}, i \in I=:\{1,2, \cdots, m\}, J_{i}(k)$ is summable for $k \in \mathbb{Z}_{0}:=\mathbb{Z} \backslash\{0\}$, and $f_{i}$ satisfy the (exponential) quasimonotone condition.

Motivated by the work [13-15, 22], in this paper, we will consider the existence of traveling wave solutions for system (1.4) with the nonlinearities $f_{i}$ satisfying the (exponential) competitive quasimonotone condition. By using the cross-iteration scheme and upper-lower solution method, we consider the 
traveling-wave solution problem for system (1.4), and give the existence result of traveling wave solutions for a delayed lattice competitive Lotka-Volterra system.

The rest of this paper is organized as follows. In Section 2, we reduce the existence of traveling wave solutions to the existence of fixed point of the operator F. In Section 3, we obtain the existence of traveling wave solutions for system (1.4). In the last section, we apply our main results to an m-dimensional delayed lattice dynamical systems with competitive interaction and prove the existence of traveling wave solutions.

\section{Preliminaries}

Throughout this paper, we employ the usual notations for the standard ordering in $\mathbb{R}^{m}$. That is, for $u=\left(u_{1}, \cdots, u_{m}\right)$ and $v=\left(v_{1}, \cdots, v_{m}\right)$, we denote $u \leqslant v$ if $u_{i} \leqslant v_{i}, i=1, \cdots, m$, and $u<v$ if $u \leqslant v$, but $u \neq v$. If $u \leqslant v$, we also denote $[u, v]=\left\{w \in \mathbb{R}^{\mathrm{m}}, u \leqslant w \leqslant v\right\}$. We use $|\cdot|$ to denote the Euclidean norm in $\mathbb{R}^{\mathrm{m}}$ and $\|\cdot\|$ to denote the supremum norm in $C\left([-\tau, 0], \mathbb{R}^{\mathrm{m}}\right)$.

A traveling wave solution of (1.4) is a special translation invariant solution of the form

$$
u_{n}^{i}(t)=\phi_{i}(n+c t), \quad i \in I=\{1,2, \cdots, m\},
$$

where $c>0$ is the wave speed and $\Phi=\left(\phi_{1}, \cdots, \phi_{m}\right)$ is the wave profile function. Substituting $u_{\mathfrak{n}}^{i}(t)=$ $\phi_{i}(n+c t)$ into (1.4) and denoting $n+c t$ still by $t$, we obtain the corresponding wave equations

$$
\begin{aligned}
c \phi_{i}^{\prime}(t)= & \sum_{k \in \mathbb{Z}_{0}} J_{i}(k)\left[\phi_{i}(t+k)-\phi_{i}(t)\right] \\
& +f_{i}\left(\sum_{l \in \mathbb{Z}} J_{i 1}(l) \phi_{1}\left(t+l-c \tau_{i 1}\right), \cdots, \sum_{l \in \mathbb{Z}} J_{i m}(l) \phi_{m}\left(t+l-c \tau_{i m}\right)\right), \quad i \in I .
\end{aligned}
$$

Note that, (2.1) is a mixed functional differential equations. For convenience, we denote

$$
\bar{f}_{i}(\Phi)(t)=f_{i}\left(\sum_{l \in \mathbb{Z}} J_{i 1}(l) \phi_{1}\left(t+l-c \tau_{i 1}\right), \cdots, \sum_{l \in \mathbb{Z}} J_{i m}(l) \phi_{m}\left(t+l-c \tau_{i m}\right)\right),
$$

then (2.1) can be reduced to

$$
c \phi_{i}^{\prime}(t)=\sum_{k \in \mathbb{Z}_{0}} J_{i}(k)\left[\phi_{i}(t+k)-\phi_{i}(t)\right]+\bar{f}_{i}(\Phi)(t), \quad i \in I .
$$

In this paper, we are only interested in traveling wave solutions satisfying the following asymptotic boundary conditions

$$
\lim _{t \rightarrow-\infty} \Phi(t)=\mathbf{0}, \quad \lim _{t \rightarrow+\infty} \Phi(t)=\mathbf{K}=:\left(k_{1}, \cdots, k_{m}\right),
$$

where $\mathbf{0}$ and $\mathbf{K}$ are two equilibria of (1.4).

Firstly, we give the (exponential) competitive quasimonotone condition (CQM or ECQM) and some assumptions for (1.4):

CQM: There exist positive constants $\beta_{i}$, such that

$$
\left\{\begin{array}{l}
f_{i}\left(\sum_{l \in \mathbb{Z}} J_{i i}(l) u_{n+l}^{i}(s), \sum_{l \in \mathbb{Z}} J_{i \tilde{i}}(l) u_{n+l}^{\tilde{i}}(s)\right)-f_{i}\left(\sum_{l \in \mathbb{Z}} J_{i i}(l) v_{n+l}^{i}(s), \sum_{l \in \mathbb{Z}} J_{i \tilde{i}}(l) u_{n+l}^{\tilde{i}}(s)\right) \\
\geqslant \sum_{k \in \mathbb{Z}_{0}} J_{i}(k)\left[u_{n}^{i}(0)-v_{n}^{i}(0)\right]-\beta_{i}\left[u_{n}^{i}(0)-v_{n}^{i}(0)\right], \\
f_{i}\left(\sum_{l \in \mathbb{Z}} J_{i i}(l) u_{n+l}^{i}(s), \sum_{l \in \mathbb{Z}} J_{i \tilde{i}}(l) u_{n+l}^{\tilde{i}}(s)\right)-f_{i}\left(\sum_{l \in \mathbb{Z}} J_{i i}(l) u_{n+l}^{i}(s), \sum_{l \in \mathbb{Z}} J_{i \tilde{i}}(l) v_{n+l}^{\tilde{i}}(s)\right) \leqslant 0,
\end{array}\right.
$$

for $v_{n}^{i}(s), u_{n}^{i}(s) \in C([-\tau, 0], \mathbb{R}), v_{n}^{i}(s) \leqslant u_{n}^{i}(s), s \in[-\tau, 0], i \in I, n \in \mathbb{Z}, \tau=\max _{i, j \in I}\left\{\tau_{i j}\right\}$, for all $\tilde{i} \in I_{i}=: I \backslash\{i\}$. 
ECQM: There exist positive constants $\beta_{i}$, such that (2.4) holds for $v_{\mathfrak{n}}^{i}(s), u_{n}^{i}(s) \in C([-\tau, 0], \mathbb{R}), i \in I, n \in$ $\mathbb{Z}, \tau=\max _{i, j \in I}\left\{\tau_{i j}\right\}$, for all $\tilde{i} \in I_{i}=: I \backslash\{i\}$ with

(i) $v_{n}^{i}(s) \leqslant u_{n}^{i}(s), s \in[-\tau, 0]$;

(ii) $e^{\beta_{i} s}\left[u_{n}^{i}(s)-v_{n}^{i}(s)\right]$ is nondecreasing for $s \in[-\tau, 0]$.

(A1) $f_{i}(0, \cdots, 0)=f_{i}\left(k_{1}, \cdots, k_{m}\right)=0$ with $k_{j}>0, i, j \in I$, and $\prod_{i=1}^{m}\left[0, M_{i}\right]\left(M_{i}>k_{i}\right)$ is a positive invariant region of the corresponding ODEs

$$
\frac{d u_{i}(t)}{d t}=f_{i}\left(u_{1}(t), \cdots, u_{m}(t)\right), \quad i \in I .
$$

(A2) There exist constants $L_{i}>0, i \in I$ such that

$$
\left|f_{i}\left(u_{1}, \cdots, u_{m}\right)-f_{i}\left(v_{1}, \cdots, v_{m}\right)\right| \leqslant L_{i}|U-V|, \quad i \in I,
$$

where $\mathbf{0} \leqslant \mathrm{U}=\left(\mathrm{u}_{1}, \cdots, \mathrm{u}_{\mathrm{m}}\right), \mathrm{V}=\left(v_{1}, \cdots, v_{\mathrm{m}}\right) \leqslant \mathbf{M}=:\left(\mathrm{M}_{1}, \cdots, \mathrm{M}_{\mathrm{m}}\right)$.

(A3) $\mathrm{J}_{\mathfrak{i}}(\mathrm{k}) \geqslant 0, k \in \mathbb{Z}_{0}$, and $0<\sum_{k \in \mathbb{Z}_{0}} \mathrm{~J}_{\mathfrak{i}}(k) e^{\lambda|k|}<+\infty, i \in I$ with $\lambda \in\left(0, \min _{i \in I}\left\{\beta_{i} / c\right\}\right)$.

(A4) $\sum_{l \in \mathbb{Z}} J_{i j}(l)=1$, and $\sum_{l \in \mathbb{Z}}\left|J_{i j}(l) e^{\lambda|l|}\right|<+\infty$, where $i, j \in I, \lambda \in\left(0, \min _{i \in I}\left\{\beta_{i} / c\right\}\right)$.

Throughout this paper, we assume that (1.4) satisfies the conditions (A1)-(A4).

Let

$$
\mathrm{C}_{[0, \mathbf{M}]}\left(\mathbb{R}, \mathbb{R}^{\mathrm{m}}\right)=\left\{\left(\phi_{1}, \cdots, \phi_{\mathrm{m}}\right) \in \mathrm{C}\left(\mathbb{R}, \mathbb{R}^{\mathrm{m}}\right): 0 \leqslant \phi_{\mathfrak{i}}(\mathrm{t}) \leqslant \mathrm{M}_{i}, i \in \mathrm{I}, \mathrm{t} \in \mathbb{R}\right\} .
$$

For $\Phi=\left(\phi_{1}, \cdots, \phi_{m}\right) \in \mathrm{C}_{[0, \mathbf{M}]}\left(\mathbb{R}, \mathbb{R}^{\mathrm{m}}\right)$, define the operator $\mathrm{H}=\left(\mathrm{H}_{1}, \cdots, \mathrm{H}_{\mathrm{m}}\right): \mathrm{C}_{[0, \mathbf{M}]}\left(\mathbb{R}, \mathbb{R}^{\mathrm{m}}\right) \rightarrow$ $\mathrm{C}\left(\mathbb{R}, \mathbb{R}^{\mathrm{m}}\right)$ by

$$
H_{i}(\Phi)(t)=\beta_{i} \phi_{i}(t)+\sum_{k \in \mathbb{Z}_{0}} J_{i}(k)\left[\phi_{i}(t+k)-\phi_{i}(t)\right]+\bar{f}_{i}(\Phi)(t)
$$

Then (2.2) can be rewritten as follows

$$
c \phi_{i}^{\prime}(t)+\beta_{i} \phi_{i}(t)-H_{i}(\Phi)(t)=0 .
$$

Furthermore, we define the operator $F=\left(F_{1}, \cdots, F_{m}\right): C_{[0, M]}\left(\mathbb{R}, \mathbb{R}^{m}\right) \rightarrow C\left(\mathbb{R}, \mathbb{R}^{m}\right)$ by

$$
F_{i}(\Phi)(t)=\frac{1}{c} e^{-\frac{\beta_{i}}{c} t} \int_{-\infty}^{t} e^{\frac{\beta_{i}}{c} s} H_{i}(\Phi)(s) d s
$$

We can easily see that $F$ is well-defined and a fixed point of $F$ is a solution of (2.1), which is a traveling wave solution of (1.4) connecting $\mathbf{0}$ with $\mathbf{K}$ if it satisfies (2.3).

For $0<\mu<\min \left\{\frac{\beta_{i}}{c}, i \in I\right\}$, we define the exponential decay norm

$$
|\Phi|_{\mu}=\sup _{t \in \mathbb{R}}|\Phi(t)| e^{-\mu|t|},
$$

for set $C\left(\mathbb{R}, \mathbb{R}^{m}\right)$. Denote

$$
\mathrm{B}_{\mu}\left(\mathbb{R}, \mathbb{R}^{\mathrm{m}}\right)=\left\{\Phi: \Phi \in \mathrm{C}\left(\mathbb{R}, \mathbb{R}^{\mathrm{m}}\right):|\Phi|_{\mu}<\infty\right\} .
$$

Then it is easy to check that $\left(B_{\mu}\left(\mathbb{R}, \mathbb{R}^{m}\right),|\cdot|_{\mu}\right)$ is a Banach space. 


\section{Existence of traveling wave solutions of system (1.4)}

In this section, we study the existence of traveling wave solutions of (1.4) when $f$ satisfies the condition CQM or ECQM respectively. Because the similarity in the verification of the following lemmas, we will give them parallelly. First, we give some nice properties of $\mathrm{H}, \mathrm{F}$ as follows.

Lemma 3.1. Assume that CQM (ECQM) holds. Then

$$
\left\{\begin{array}{l}
H_{1}\left(\phi_{1}, \psi_{2}, \psi_{3}, \cdots, \psi_{m}\right)(t) \geqslant H_{1}\left(\psi_{1}, \phi_{2}, \phi_{3}, \cdots, \phi_{m}\right)(t), \\
H_{2}\left(\psi_{1}, \phi_{2}, \psi_{3}, \cdots, \psi_{m}\right)(t) \geqslant H_{2}\left(\phi_{1}, \psi_{2}, \phi_{3}, \cdots, \phi_{m}\right)(t), \\
\quad \vdots \\
H_{m}\left(\psi_{1}, \psi_{2}, \psi_{3}, \cdots, \phi_{m}\right)(t) \geqslant H_{m}\left(\phi_{1}, \phi_{2}, \phi_{3}, \cdots, \psi_{m}\right)(t),
\end{array}\right.
$$

and

$$
\left\{\begin{array}{l}
F_{1}\left(\phi_{1}, \psi_{2}, \psi_{3}, \cdots, \psi_{m}\right)(t) \geqslant F_{1}\left(\psi_{1}, \phi_{2}, \phi_{3}, \cdots, \phi_{m}\right)(t) \\
F_{2}\left(\psi_{1}, \phi_{2}, \psi_{3}, \cdots, \psi_{m}\right)(t) \geqslant F_{2}\left(\phi_{1}, \psi_{2}, \phi_{3}, \cdots, \phi_{m}\right)(t) \\
\quad \vdots \\
F_{m}\left(\psi_{1}, \psi_{2}, \psi_{3}, \cdots, \phi_{m}\right)(t) \geqslant F_{m}\left(\phi_{1}, \phi_{2}, \phi_{3}, \cdots, \psi_{m}\right)(t)
\end{array}\right.
$$

for $\Phi=\left(\phi_{1}, \cdots, \phi_{\mathrm{m}}\right), \Psi=\left(\psi_{1}, \cdots, \psi_{\mathrm{m}}\right) \in \mathrm{C}\left(\mathbb{R}, \mathbb{R}^{\mathrm{m}}\right)$ with

$$
\left\{\begin{array}{l}
\left(\text { i) } 0 \leqslant \psi_{i}(t) \leqslant \phi_{i}(t) \leqslant M_{i}, t \in \mathbb{R}, i \in I\right. \\
\left(\text { ii) } e^{\frac{\beta_{i}}{c} t}\left[\phi_{i}(t)-\psi_{i}(t)\right] \text { is nondecreasing for } t \in \mathbb{R} .\right.
\end{array}\right.
$$

Proof. We only prove it when $f$ satisfies ECQM condition. According to ECQM, (A3) and the definition of operator $\mathrm{H}$, we have

$$
\begin{aligned}
& \mathrm{H}_{1}\left(\phi_{1}, \psi_{2}, \psi_{3}, \cdots, \psi_{\mathrm{m}}\right)(\mathrm{t})-\mathrm{H}_{1}\left(\psi_{1}, \phi_{2}, \phi_{3}, \cdots, \phi_{\mathrm{m}}\right)(\mathrm{t}) \\
&= \mathrm{H}_{1}\left(\phi_{1}, \psi_{2}, \psi_{3}, \cdots, \psi_{\mathrm{m}}\right)(\mathrm{t}) \\
&-\mathrm{H}_{1}\left(\psi_{1}, \psi_{2}, \psi_{3}, \cdots, \psi_{\mathrm{m}}\right)(\mathrm{t}) \\
&+\mathrm{H}_{1}\left(\psi_{1}, \psi_{2}, \psi_{3}, \cdots, \psi_{\mathrm{m}}\right)(\mathrm{t}) \\
&-\mathrm{H}_{1}\left(\psi_{1}, \phi_{2}, \phi_{3}, \cdots, \phi_{\mathrm{m}}\right)(\mathrm{t}) \\
&= \beta_{1}\left(\phi_{1}(\mathrm{t})-\psi_{1}(\mathrm{t})\right) \\
&-\sum_{k \in \mathbb{Z}_{0}} \mathrm{~J}_{1}(\mathrm{k})\left(\phi_{1}(\mathrm{t})-\psi_{1}(\mathrm{t})\right) \\
&+\overline{\mathrm{f}}_{1}\left(\phi_{1}, \psi_{2}, \cdots, \psi_{\mathrm{m}}\right)(\mathrm{t})-\overline{\mathrm{f}}_{1}\left(\psi_{1}, \psi_{2}, \cdots, \psi_{\mathrm{m}}\right)(\mathrm{t}) \\
&+\overline{\mathrm{f}}_{1}\left(\psi_{1}, \psi_{2}, \cdots, \psi_{\mathrm{m}}\right)(\mathrm{t})-\overline{\mathrm{f}}_{1}\left(\psi_{1}, \phi_{2}, \cdots, \phi_{\mathrm{m}}\right)(\mathrm{t}) \\
&+\sum_{\mathrm{k} \in \mathbb{Z}_{0}} \mathrm{~J}_{1}(\mathrm{k})\left(\left(\phi_{1}(\mathrm{t}+\mathrm{k})-\psi_{1}(\mathrm{t}+\mathrm{k})\right)\right. \\
& \geqslant \sum_{\mathrm{k} \in \mathbb{Z}_{0}} \mathrm{~J}_{1}(\mathrm{k})\left(\phi_{1}(\mathrm{t}+\mathrm{k})-\psi_{1}(\mathrm{t}+\mathrm{k})\right) \geqslant 0 .
\end{aligned}
$$

It follows from (2.5) and property of $\mathrm{H}_{1}$ that

$$
F_{1}\left(\phi_{1}, \psi_{2}, \psi_{3}, \cdots, \psi_{m}\right)(t) \geqslant F_{1}\left(\psi_{1}, \phi_{2}, \phi_{3}, \cdots, \phi_{m}\right)(t) .
$$

The other inequalities can be verified in the same method. This completes the proof. 
Similar to $[14$, Lemmas $3.5,3.6]$, we have the following.

\section{Lemma 3.2.}

$$
\mathrm{F}=\left(\mathrm{F}_{1}, \cdots, \mathrm{F}_{\mathrm{m}}\right): \mathrm{C}_{[0, \mathbf{M}]}\left(\mathbb{R}, \mathbb{R}^{\mathrm{m}}\right) \rightarrow \mathrm{C}\left(\mathbb{R}, \mathbb{R}^{\mathrm{m}}\right),
$$

is continuous and compact with respect to the decay norm $|\cdot|_{\mu}$.

Nxet, we assume that (2.1) has a pair of upper and lower solutions as below.

Definition 3.3. A pair of continuous functions $\bar{\Phi}=\left(\bar{\phi}_{1}, \cdots, \bar{\phi}_{\mathrm{m}}\right)$ and $\underline{\Phi}=\left(\underline{\phi}_{1}, \cdots, \underline{\phi}_{\mathrm{m}}\right)$ are called an upper solution and a lower solution of (2.1), respectively, if $\bar{\Phi}$ and $\Phi$ are continuous differentiable in $\mathbb{R} \backslash \mathbb{T}$ and satisfy

$$
\begin{aligned}
& c \bar{\phi}_{i}^{\prime}(t) \geqslant \sum_{k \in \mathbb{Z}} J_{i}(k)\left[\bar{\phi}_{i}(t+k)-\bar{\phi}_{i}(t)\right]+\bar{f}_{i}\left(\underline{\phi}_{1}, \cdots, \underline{\phi}_{i-1}, \bar{\phi}_{i}, \underline{\phi}_{i+1}, \cdots, \underline{\phi}_{m}\right)(t), \\
& c \underline{\phi}_{i}^{\prime}(t) \leqslant \sum_{k \in \mathbb{Z}_{0}} J_{i}(k)\left[\underline{\phi}_{i}(t+k)-\underline{\phi}_{i}(t)\right]+\bar{f}_{i}\left(\bar{\phi}_{1}, \cdots, \bar{\phi}_{i-1}, \underline{\phi}_{i}, \bar{\phi}_{i+1}, \cdots, \bar{\phi}_{m}\right)(t),
\end{aligned}
$$

for $i \in I, t \in \mathbb{R} \backslash \mathbb{T}$, where $\mathbb{T}=\left\{T_{1}, T_{2}, \cdots, T_{s}\right\}$ with $T_{1}<T_{2}<\cdots<T_{s}$.

Furthermore, we give some hypotheses (P1)-(P3):

(P1) $0 \leqslant\left(\underline{\phi}_{1}, \cdots, \underline{\phi}_{m}\right) \leqslant\left(\bar{\phi}_{1}, \cdots, \bar{\phi}_{m}\right) \leqslant \mathbf{M} ;$

(P2) $\lim _{\mathrm{t} \rightarrow-\infty}\left(\bar{\phi}_{1}, \cdots, \bar{\phi}_{\mathrm{m}}\right)=\mathbf{0}, \lim _{\mathrm{t} \rightarrow+\infty}\left(\underline{\phi}_{1}, \cdots, \underline{\phi}_{\mathrm{m}}\right)=\lim _{\mathrm{t} \rightarrow+\infty}\left(\bar{\phi}_{1}, \cdots, \bar{\phi}_{\mathrm{m}}\right)=\mathbf{K}$;

(P3) $e^{\frac{\beta_{i}}{c} t}\left[\bar{\phi}_{i}(t)-\underline{\phi}_{i}(t)\right], i \in I$ are nondecreasing for $t \in \mathbb{R}$.

We define the wave profile set

$$
\Gamma:=\left\{\Phi=\left(\phi_{1}, \cdots, \phi_{m}\right) \in \mathrm{C}_{[0, \mathbf{M}]}\left(\mathbb{R}, \mathbb{R}^{\mathrm{m}}\right):\left(\underline{\phi}_{1}, \cdots, \underline{\phi}_{\mathrm{m}}\right) \leqslant\left(\phi_{1}, \cdots, \phi_{\mathrm{m}}\right) \leqslant\left(\bar{\phi}_{1}, \cdots, \bar{\phi}_{\mathrm{m}}\right)\right\},
$$

when the upper-lower solutions satisfy hypotheses (P1)-(P2), and $\Gamma^{*}$ by

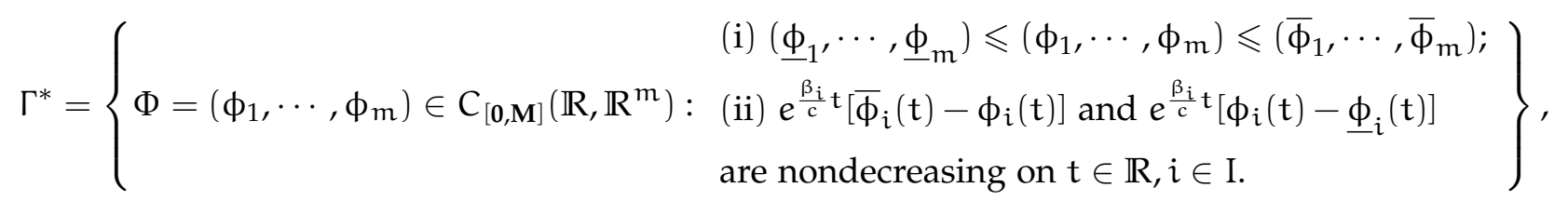

when the upper-lower solutions satisfy hypotheses (P1)-(P3), respectively. It is easy to see that $\Gamma$ and $\Gamma^{*}$ are non-empty, closed and bounded convex subsets of $B_{\mu}\left(\mathbb{R}, \mathbb{R}^{m}\right)$.

Lemma 3.4. Assume that CQM and (P1)-(P2) hold, then $\mathrm{F}(\Gamma) \subset \Gamma$.

Lemma 3.5. Assume that ECQM and (P1)-(P3) hold, then $\mathrm{F}\left(\Gamma^{*}\right) \subset \Gamma^{*}$.

Proof. First of all, we prove that $\mathrm{F}(\Phi)$ satisfies (i) of $\Gamma^{*}$.

For any $\Phi=\left(\phi_{1}, \cdots, \phi_{m}\right) \in \Gamma^{*}$, by Lemma 3.1, it suffices to verify that

$$
\left\{\begin{array}{l}
\underline{\phi}_{1}(t) \leqslant F_{1}\left(\underline{\phi}_{1}, \bar{\phi}_{2}, \cdots, \bar{\phi}_{m}\right)(t) \leqslant F_{1}\left(\bar{\phi}_{1}, \phi_{2}, \cdots, \underline{\phi}_{m}\right)(t) \leqslant \bar{\phi}_{1}(t) \\
\underline{\phi}_{2}(t) \leqslant F_{2}\left(\bar{\phi}_{1}, \underline{\phi}_{2}, \cdots, \bar{\phi}_{m}\right)(t) \leqslant F_{2}\left(\underline{\phi}_{1}, \bar{\phi}_{2}, \cdots, \underline{\phi}_{m}\right)(t) \leqslant \bar{\phi}_{2}(t) \\
\quad \vdots \\
\underline{\phi}_{m}(t) \leqslant F_{m}\left(\bar{\phi}_{1}, \cdots, \bar{\phi}_{m-1}, \underline{\phi}_{m}\right)(t) \leqslant F_{m}\left(\underline{\phi}_{1}, \cdots, \underline{\phi}_{m-1}, \bar{\phi}_{m}\right)(t) \leqslant \bar{\phi}_{m}(t)
\end{array}\right.
$$


According to the definitions of upper and lower solutions, we obtain

$$
c \bar{\phi}_{i}^{\prime}(t)+\beta_{i} \bar{\phi}_{i}(t)-H_{i}\left(\underline{\phi}_{1}, \cdots, \underline{\phi}_{i-1}, \bar{\phi}_{i}, \underline{\phi}_{i+1}, \cdots, \underline{\phi}_{m}\right)(t) \geqslant 0,
$$

for $t \in \mathbb{R} \backslash \mathbb{T}, i \in \mathrm{I}$.

Let $\mathrm{T}_{0}=-\infty$ and $\mathrm{T}_{\mathrm{s}+1}=+\infty$. Then

$$
\begin{aligned}
\mathrm{F}_{1}\left(\bar{\phi}_{1}, \underline{\phi}_{2}, \cdots, \underline{\phi}_{\mathrm{m}}\right)(\mathrm{t}) & =\frac{1}{\mathrm{c}} \mathrm{e}^{-\frac{\beta_{1}}{\mathrm{c}} \mathrm{t}} \int_{-\infty}^{\mathrm{t}} \mathrm{e}^{\frac{\beta_{1}}{\mathrm{c}} \mathrm{s}} \mathrm{H}_{1}\left(\bar{\phi}_{1}, \underline{\phi}_{2}, \cdots, \underline{\phi}_{\mathrm{m}}\right)(\mathrm{s}) \mathrm{d} \mathrm{s} \\
& \leqslant \frac{1}{\mathrm{c}} e^{-\frac{\beta_{1}}{\mathrm{c}} \mathrm{t}}\left(\sum_{j=1}^{\mathrm{i}-1} \int_{\mathrm{T}_{j-1}}^{\mathrm{T}_{j}}+\int_{\mathrm{T}_{i-1}}^{\mathrm{t}}\right) \mathrm{e}^{\frac{\beta_{1}}{\mathrm{c}} \mathrm{s}}\left[\mathrm{c} \bar{\phi}_{1}^{\prime}(\mathrm{s})+\beta_{1} \bar{\phi}_{1}(\mathrm{~s})\right] \mathrm{d} \mathrm{s} \\
& =\bar{\phi}_{1}(\mathrm{t}), \quad \mathrm{T}_{\mathrm{i}-1}<\mathrm{t}<\mathrm{T}_{\mathrm{i}},
\end{aligned}
$$

where $i=1,2, \cdots, s+1$, and the continuity of $F_{1}\left(\bar{\phi}_{1}, \underline{\phi}_{2}, \cdots, \underline{\phi}_{m}\right)(t)$ and $\bar{\phi}_{1}(t)$ implies

$$
\mathrm{F}_{1}\left(\bar{\phi}_{1}, \underline{\phi}_{2}, \cdots, \underline{\phi}_{\mathrm{m}}\right)(\mathrm{t}) \leqslant \bar{\phi}_{1}(\mathrm{t})
$$

for all $t \in \mathbb{R}$. By a similar argument, the remainders of (3.1) are also valid.

Next we need to verify the condition (ii) of $\Gamma^{*}$. According to the definition of $F$, we have

$$
e^{\frac{\beta_{i}}{c} t}\left[\bar{\phi}_{\mathfrak{i}}(t)-F_{i}\left(\phi_{1}, \cdots, \phi_{m}\right)(t)\right]=e^{\frac{\beta_{i}}{c} t} \bar{\phi}_{i}(t)-\frac{1}{c} \int_{-\infty}^{t} e^{\frac{\beta_{i}}{c} s} H_{i}\left(\phi_{1}, \cdots, \phi_{m}\right)(s) d s,
$$

then,

$$
\begin{aligned}
\frac{\mathrm{d}}{\mathrm{dt}}\left\{e ^ { \frac { \beta _ { i } } { \mathrm { c } } \mathrm { t } } \left[\bar{\phi}_{i}(\mathrm{t})-\right.\right. & \left.\left.F_{i}\left(\phi_{1}, \cdots, \phi_{m}\right)(t)\right]\right\} \\
& =e^{\frac{\beta_{i}}{c} t}\left(\frac{\beta_{i}}{c} \bar{\phi}_{i}(t)+\bar{\phi}_{i}^{\prime}(t)\right)-\frac{1}{c} e^{\frac{\beta_{i}}{c} t} H_{i}\left(\phi_{1}, \cdots, \phi_{m}\right)(t) \\
& =\frac{1}{c} e^{\frac{\beta_{i}}{c} t}\left[c \bar{\phi}_{i}^{\prime}(t)+\beta_{i} \bar{\phi}_{i}(t)-H_{i}\left(\phi_{1}, \cdots, \phi_{m}\right)(t)\right] \\
& \geqslant \frac{1}{c} e^{\frac{\beta_{i}}{c} t}\left[H_{i}\left(\underline{\phi}_{1}, \cdots, \underline{\phi}_{i-1}, \bar{\phi}_{i}, \underline{\phi}_{i+1}, \cdots, \underline{\phi}_{m}\right)(t)-H_{i}\left(\phi_{1}, \cdots, \phi_{m}\right)(t)\right] \\
& \geqslant 0, \quad t \in \mathbb{R} \backslash \mathbb{T} .
\end{aligned}
$$

By continuity of $F_{i}\left(\phi_{1}, \phi_{2}, \cdots, \phi_{m}\right)(t)$ and $\bar{\phi}_{i}(t)$, we see that $e^{\frac{\beta_{i}}{c} t}\left[\bar{\phi}_{i}(t)-F_{i}\left(\phi_{1}, \cdots, \phi_{m}\right)(t)\right]$ is nondecreasing in $t \in \mathbb{R}$. Also, we can similarly prove that $e^{\frac{\beta_{i}}{c} t}\left[F_{i}\left(\phi_{1}, \cdots, \phi_{m}\right)(t)-\underline{\phi}_{i}(t)\right]$ is nondecreasing in $t \in \mathbb{R}$. This completes the proof.

Now, we are in a position to state the following main theorem.

Theorem 3.6. Assume CQM holds, and further that (2.1) has a pair of upper-lower solution $\bar{\Phi}=\left(\bar{\phi}_{1}, \ldots, \bar{\phi}_{m}\right)$, $\underline{\Phi}=\left(\underline{\phi}_{1}, \cdots, \underline{\phi}_{m}\right)$ satisfying (P1)-(P2). Then (1.4) has a traveling wave solution satisfying (2.3).

Theorem 3.7. Assume that ECQM holds, and further that (2.1) has a pair of upper-lower solution $\bar{\Phi}=\left(\bar{\phi}_{1}, \cdots, \bar{\phi}_{m}\right), \underline{\Phi}=\left(\underline{\phi}_{1}, \cdots, \underline{\Phi}_{m}\right)$ satisfying (P1)-(P3). Then (1.4) has a traveling wave solution satisfying (2.3).

Proof. From Lemmas 3.2 and 3.5, we know that $F\left(\Gamma^{*}\right) \subset \Gamma^{*}$ and $F$ is compact. By the Schauder's fixed point theorem, there exists a fixed point $\left(\phi_{1}^{*}, \cdots, \phi_{m}^{*}\right) \in \Gamma^{*}$, which is a solution of $(2.1)$, that is a traveling wave solution of (1.4). 
Next, we verify the boundary conditions (2.3).

By (P2) and the inequality

$$
0 \leqslant\left(\underline{\phi}_{1}, \cdots, \underline{\phi}_{m}\right) \leqslant\left(\phi_{1}^{*}, \cdots, \phi_{m}^{*}\right) \leqslant\left(\bar{\phi}_{1}, \cdots, \bar{\phi}_{m}\right) \leqslant\left(M_{1}, \cdots, M_{m}\right),
$$

we see that

$$
\lim _{t \rightarrow-\infty}\left(\phi_{1}^{*}(t), \cdots, \phi_{m}^{*}(t)\right)=(0, \cdots, 0), \lim _{t \rightarrow+\infty}\left(\phi_{1}^{*}(t), \cdots, \phi_{m}^{*}(t)\right)=\left(k_{1}, \cdots, k_{m}\right) .
$$

Therefore, the fixed point $\left(\phi_{1}^{*}(t), \cdots, \phi_{m}^{*}(t)\right)$ satisfies the boundary conditions (2.3). The proof is complete.

\section{Applications}

In this section, we employ our conclusions to establish the existence of traveling wave solutions for the following system.

Example 4.1. Consider the following m-dimensional delayed lattice dynamical systems with CQM condition and global interaction

$$
\left\{\begin{array}{l}
\frac{d u_{n}^{1}(t)}{d t}=\sum_{k \in \mathbb{Z}_{0}} J_{1}(k)\left[u_{n+k}^{1}(t)-u_{n}^{1}(t)\right]+r_{1} u_{n}^{1}(t)\left[1-a_{11} u_{n}^{1}(t)-\sum_{j \in I_{1}} a_{1 j} u_{n}^{j}\left(t-\tau_{1 j}\right)\right], \\
\vdots \\
\frac{d u_{n}^{m}(t)}{d t}=\sum_{k \in \mathbb{Z}_{0}} J_{m}(k)\left[u_{n+k}^{m}(t)-u_{n}^{m}(t)\right]+r_{m} u_{n}^{m}(t)\left[1-\sum_{j \in I_{m}} a_{m j} u_{n}^{j}\left(t-\tau_{m j}\right)-a_{m m} u_{n}^{m}(t)\right],
\end{array}\right.
$$

where $r_{i}>0, a_{i j}>0, J_{i}(k), i \in I$ satisfy condition (A3), $I_{i}=I \backslash\{i\}$. (4.1) has a trivial steady state $E_{0}=$ $(0,0, \cdots, 0)$. Moreover, we assume that there exists $E^{*}=\left(k_{1}, k_{2}, \cdots, k_{m}\right), k_{i}>0$ such that $\sum_{j \in I} a_{i j} k_{j}=1$, and

$$
a_{i i} k_{i}>\sum_{j \in I_{i}} a_{i j} k_{j}, \quad i \in I .
$$

Thus, we are interested with the existence of traveling wave solutions of (4.1) connecting $E_{0}$ and $E^{*}$. Let $\mathbf{M}=\left(M_{1}, \cdots, M_{m}\right)$ with $M_{i}=\frac{1}{a_{i i}}$, then $[\mathbf{0}, \mathbf{M}]$ is an invariant region of the corresponding ODEs.

Substituting $u_{n}^{i}(t)=\phi_{i}(n+c t)$ into (4.1) and denoting $n+c t$ still by $t$, we derive the corresponding wave equations

$$
\left\{\begin{array}{l}
c \phi_{1}^{\prime}(t)=\sum_{k \in \mathbb{Z}_{0}} J_{1}(k)\left[\phi_{1}(t+k)-\phi_{1}(t)\right]+r_{1} \phi_{1}(t)\left[1-a_{11} \phi_{1}(t)-\sum_{j \in I_{1}} a_{1 j} \phi_{m}\left(t-c \tau_{1 j}\right)\right], \\
\vdots \\
c \phi_{m}^{\prime}(t)=\sum_{k \in \mathbb{Z}_{0}} J_{m}(k)\left[\phi_{m}(t+k)-\phi_{m}(t)\right]+r_{m} \phi_{m}(t)\left[1-\sum_{j \in I_{m}} a_{m j} \phi_{m}\left(t-c \tau_{1 j}\right)-a_{m m} \phi_{m}(t)\right] .
\end{array}\right.
$$

For $\phi_{1}, \cdots, \phi_{m} \in \mathrm{C}([-\tau, 0], \mathbb{R})$, denote

$$
\begin{aligned}
& f_{1}\left(\phi_{1}, \cdots, \phi_{m}\right)=r_{1} \phi_{1}(0)\left[1-a_{11} \phi_{1}(0)-\sum_{j \in I_{1}} a_{1 j} \phi_{m}\left(t-\tau_{1 j}\right)\right] \\
& \vdots \\
& f_{m}\left(\phi_{1}, \cdots, \phi_{m}\right)=r_{m} \phi_{m}(0)\left[1-\sum_{j \in I_{1}} a_{m j} \phi_{m}\left(t-\tau_{m j}\right)-a_{m m} \phi_{m}(0)\right] .
\end{aligned}
$$

Obviously, $f=\left(f_{1}, \cdots, f_{m}\right)$ satisfies assumptions (A1), (A2) and CQM condition. 
In order to apply Theorem 3.6, we need to construct an upper solution and a lower solution for (4.3). For $c \geqslant 0, \lambda \geqslant 0$, we define

$$
\Delta_{i}(\lambda, c)=\sum_{k \in \mathbb{Z}_{0}} J_{i}(k)\left(e^{\lambda k}-1\right)-c \lambda+r_{i}
$$

Then $\Delta_{\mathfrak{i}}(\lambda, c)$ is well-defined. Furthermore, we have the following lemma.

Lemma 4.2. There exist $c_{i}>0, i \in I$ such that the following hold:

if $\mathrm{c}>\mathrm{c}_{\mathrm{i}}, \Delta_{\mathrm{i}}(\lambda, \mathrm{c})$ has two distinct positive roots $\lambda_{\mathrm{i} 1}<\lambda_{\mathrm{i} 2}$, and

$$
\Delta_{i}(\lambda, c)= \begin{cases}>0, & \lambda \in\left(0, \lambda_{i 1}\right) \cup\left(\lambda_{i 2}, \infty\right), \\ <0, & \lambda \in\left(\lambda_{i 1}, \lambda_{i 2}\right),\end{cases}
$$

if $\mathrm{c}<\mathrm{c}_{\mathfrak{i}}, \Delta_{\mathfrak{i}}(\lambda, \mathrm{c})$ has no positive root.

Let $c^{*}=\max \left\{c_{1}, c_{2}, \cdots, c_{m}\right\}$, we always assume that $c>c^{*}$. Assume that $q>1$ holds and $\eta$ satisfies

$$
\eta \in\left(1, \min _{i, j \in I}\left\{\frac{\lambda_{i 2}}{\lambda_{i 1}}, \frac{\lambda_{i 1}+\lambda_{j 1}}{\lambda_{i 1}}\right\}\right)
$$

Define $l_{i}(t)=e^{\lambda_{i 1} t}-q e^{\eta \lambda_{i 1} t}, i \in I$. Noting that $l_{i}(t)$ are unimodal functions, and by careful calculation, we can derive that $l_{i}(t), i \in I$ have a common maximum expressed by

$$
\sigma=(\eta-1) \eta^{\frac{\eta}{1-\eta}} q^{\frac{1}{1-\eta}}>0,
$$

which correct the inappropriate statement in [13, 22], and can simplify the verification of upper-lower solution for system (4.3).

Furthermore, we denote constants $t_{i 3}$ such that

$$
t_{i 3}=\max \left\{t: l_{i}(t)=\frac{\sigma}{2}\right\} .
$$

Let $\lambda \in(0,1)$ be small enough such that

$$
k_{i}-\left(k_{i}-\frac{\sigma}{2}\right) e^{-\lambda t_{i 3}} \geqslant \frac{\sigma}{4}
$$

Then, for every $i \in I$, we can define the continuous function $\underline{\Phi}(t)=\left(\underline{\phi}_{1}(t), \cdots, \underline{\phi}_{m}(t)\right)$ by

$$
\underline{\Phi}_{i}(t)= \begin{cases}e^{\lambda_{i 1} t}-q e^{\eta \lambda_{i 1} t}, & t \leqslant t_{i 2} \\ k_{i}-\left(k_{i}-\frac{\sigma}{2}\right) e^{-\lambda t}, & t \geqslant t_{i 2}\end{cases}
$$

where $t_{i 2}>t_{i 3}$ for all $i \in I$.

Remark 4.3. By calculating, we can conclude that $t_{i 2}$ is dependent on parameter $q$. Thus, we can choose $q$ large enough such that $t_{i 2}<0$, which is crucial in the verifications of upper-lower solutions.

Define the continuous function $\bar{\Phi}(\mathrm{t})=\left(\bar{\phi}_{1}(\mathrm{t}), \cdots, \bar{\phi}_{\mathrm{m}}(\mathrm{t})\right)$ as follows:

$$
\bar{\phi}_{i}(t)= \begin{cases}\min \left\{M_{i}, e^{\lambda_{i 1} t}\right\}, & t \leqslant t_{i 1}, \\ k_{i}+k_{i} e^{-\lambda t}, & t \geqslant t_{i 1} .\end{cases}
$$

Remark 4.4. In view of (4.2), there exists $\bar{\epsilon}_{i} \in\left(0, k_{i}\right)$ such that

$$
a_{i i} k_{i}>\sum_{j \in I_{i}} a_{i j} \epsilon_{j}, \quad a_{i i} \epsilon_{i}>\sum_{j \in I_{i}} a_{i j} k_{j},
$$

hold for any $\epsilon_{i} \in\left[\bar{\epsilon}_{i}, k_{i}\right]$, which are useful in the following demonstrations. 
Lemma 4.5. For $\mathrm{q}>1$ being large enough, the following statements

$$
\begin{aligned}
& q>\max _{i \in I}\left\{\frac{r_{i} \sum_{j \in I} a_{i j}}{-\Delta_{i}\left(\eta \lambda_{i 1}, c\right)}+1\right\}, \\
& k_{i}-\bar{\epsilon}_{i}>\frac{\sigma}{2}, \\
& \min _{i \in I}\left\{t_{i 1}\right\} \geqslant \max _{i \in I}\left\{t_{i 2}\right\}+c \tau .
\end{aligned}
$$

are valid.

Proof. By Lemma 4.2 and (4.4), we have (4.8) holds. From (4.5), we know that $\sigma$ is decreasing with respect to $q$. Then, we can choose q large enough such that (4.9) holds. For (4.10), we have $t_{i 1} \geqslant \frac{\ln k_{i}}{\lambda_{i}}$. Since $\phi_{i}(t), i \in I$ are continuous functions, we obtain that

$$
q e^{\eta \lambda_{i 1} t_{i 2}}=e^{\lambda_{i 1} t_{i 2}}-k_{i}+\left(k_{i}-\frac{\sigma}{2}\right) e^{-\lambda t_{i 2}}:=N_{i} .
$$

Thus, $t_{i 2}=\frac{1}{\eta \lambda_{i 1}}\left(\ln N_{i}-\ln q\right)$. Therefore, there exists $q>1$ sufficiently large such that (4.10) holds.

It is easy to see that $\bar{\Phi}(\mathrm{t}), \underline{\Phi}(\mathrm{t})$ satisfy $(\mathrm{P} 1),(\mathrm{P} 2)$. We now prove that $\bar{\Phi}(\mathrm{t})$ and $\underline{\Phi}(\mathrm{t})$ are an upper solution and a lower solution of (4.3), respectively.

Lemma 4.6. Assume that (4.2) and (4.4) hold. Then $\left(\bar{\phi}_{1}(\mathrm{t}), \bar{\phi}_{2}(\mathrm{t}), \ldots, \bar{\phi}_{\mathrm{m}}(\mathrm{t})\right)$ is an upper solution of (4.3).

Proof. For $\bar{\phi}_{1}(t)$, it suffices to prove that

$$
\overline{\mathrm{L}}_{1}(\mathrm{t}):=\sum_{\mathrm{k} \in \mathbb{Z}_{0}} J_{1}(\mathrm{k})\left[\bar{\phi}_{1}(\mathrm{t}+\mathrm{k})-\bar{\phi}_{1}(\mathrm{t})\right]-\mathrm{c} \bar{\phi}_{1}^{\prime}(\mathrm{t})+\mathrm{r}_{1} \bar{\phi}_{1}(\mathrm{t})\left[1-\mathrm{a}_{11} \bar{\phi}_{1}(\mathrm{t})-\sum_{\mathrm{j} \in \mathrm{I}_{1}} \mathrm{a}_{1 j} \underline{\phi}_{\mathrm{j}}\left(\mathrm{t}-\mathrm{c} \tau_{1 j}\right)\right] \leqslant 0 .
$$

When $t \leqslant t_{11}$, noting that for any $k \in \mathbb{Z}_{0}$,

$$
\bar{\phi}_{1}(t+k) \leqslant M_{1}\left(=k_{1}+k_{1} e^{-\lambda t_{11}}\right),
$$

equation (4.11) is clear if $\bar{\phi}_{1}(t)=M_{1}$.

If $\bar{\phi}_{1}(t)=e^{\lambda_{11} t}$, noting that for any $k \in \mathbb{Z}_{0}, \bar{\phi}_{1}(t+k) \leqslant e^{\lambda_{11}(t+k)}$ and $\underline{\phi}_{j}\left(t-c \tau_{1 j}\right)>0, j \in I_{1}$, we have

$$
\begin{aligned}
\overline{\mathrm{L}}_{1}(\mathrm{t}) & \leqslant \sum_{\mathrm{k} \in \mathbb{Z}_{0}} \mathrm{~J}_{1}(\mathrm{k})\left[\bar{\phi}_{1}(\mathrm{t}+\mathrm{k})-\bar{\phi}_{1}(\mathrm{t})\right]-\mathrm{c} \bar{\phi}_{1}^{\prime}(\mathrm{t})+\mathrm{r}_{1} \bar{\phi}_{1}(\mathrm{t}) \\
& \leqslant \mathrm{e}^{\lambda_{11} \mathrm{t}}\left[\sum_{\mathrm{k} \in \mathbb{Z}_{0}}\left(\mathrm{e}^{\lambda_{11} \mathrm{k}}-1\right)-\mathrm{c} \lambda_{11}+\mathrm{r}_{1}\right]=0 .
\end{aligned}
$$

When $t>t_{11}$, then $\bar{\phi}_{1}(t)=k_{1}+k_{1} e^{-\lambda t}$. Due to (4.10), we have $\underline{\phi}_{j}\left(t-c \tau_{1 j}\right)=k_{j}-\left(k_{j}-\frac{\sigma}{2}\right) e^{-\lambda\left(t-c \tau_{1 j}\right)}$, $j \in I_{1}$. By (4.12), we have

$$
\begin{aligned}
\overline{\mathrm{L}}_{1}(\mathrm{t}) \leqslant & \sum_{k \in \mathbb{Z}_{0}} \mathrm{~J}_{1}(k)\left[k_{1}+k_{1} e^{-\lambda \mathrm{t}_{11}}-\left(k_{1}+k_{1} e^{-\lambda t}\right)\right]+c \lambda k_{1} e^{-\lambda t}+r_{1}\left(k_{1}+k_{1} e^{-\lambda t}\right) \\
& {\left[1-a_{11}\left(k_{1}+k_{1} e^{-\lambda t}\right)-\sum_{j \in I_{1}} a_{1 j}\left(k_{j}-\left(k_{j}-\frac{\sigma}{2}\right)\right) e^{-\lambda\left(t-c \tau_{1 j}\right)}\right] } \\
\leqslant & k_{1} e^{-\lambda t}\left\{\sum_{k \in \mathbb{Z}_{0}} J_{1}(k)\left[e^{-\lambda\left(t_{11}-t\right)}-1\right]+c \lambda+r_{1}\left(1+e^{-\lambda t}\right)\right.
\end{aligned}
$$




$$
\begin{aligned}
& {\left.\left[\sum_{j \in I_{1}} a_{1 j}\left(k_{j}-\frac{\sigma}{2}\right) e^{\lambda c \tau_{1 j}}-a_{11} k_{1}\right]\right\} } \\
= & : k_{1} e^{-\lambda t} I_{1}(\lambda) .
\end{aligned}
$$

Since

$$
I_{1}(0)=2 r_{1}\left[\sum_{j \in I_{1}} a_{1 j}\left(k_{j}-\frac{\sigma}{2}\right)-a_{11} k_{1}\right]<0,
$$

then there exists $\lambda_{1}^{*}>0$ such that $I_{1}(\lambda)<0$ for any $\lambda \in\left(0, \lambda_{1}^{*}\right)$. Thus, $\bar{L}_{1}(t) \leqslant 0$ holds for $\lambda \in\left(0, \lambda_{1}^{*}\right)$ if $t>t_{11}$.

Similarly, we can prove that

$$
\sum_{k \in \mathbb{Z}_{0}} J_{i}(k)\left[\bar{\phi}_{i}(t+k)-\bar{\phi}_{i}(t)\right]-c \bar{\phi}_{i}^{\prime}(t)+r_{i} \bar{\phi}_{i}(t)\left[1-a_{i i} \bar{\phi}_{i}(t)-\sum_{j \in I_{i}} a_{i j} \underline{\phi}_{j}\left(t-c \tau_{i j}\right)\right] \leqslant 0,
$$

for $i=2, \cdots, m$.

By the above argument, we see that $\bar{\phi}_{1}(t), \bar{\phi}_{2}(t), \cdots, \bar{\phi}_{m}(t)$ is an upper solution of (4.3).

Lemma 4.7. Assume that (4.2) and (4.4) hold. Then $\left(\underline{\phi}_{1}(\mathrm{t}), \underline{\phi}_{2}(\mathrm{t}), \cdots, \underline{\phi}_{\mathrm{m}}(\mathrm{t})\right)$ is a lower solution of (4.3).

Proof. For $\phi_{1}(t)$, it suffices to prove that

$$
\underline{L}_{1}(t):=\sum_{k \in \mathbb{Z}_{0}} J_{1}(k)\left[\underline{\phi}_{1}(t+k)-\underline{\phi}_{1}(t)\right]-c \underline{\phi}_{1}^{\prime}(t)+r_{1} \underline{\phi}_{1}(t)\left[1-a_{11} \underline{\phi}_{1}(t)-\sum_{j \in I_{1}} a_{1 j} \bar{\phi}_{j}\left(t-c \tau_{1 j}\right)\right] \geqslant 0 .
$$

When $t \leqslant t_{12}$, then $\underline{\phi}_{1}(t)=e^{\lambda_{11} t}-q e^{\eta \lambda_{11} t}, \bar{\phi}_{j}\left(t-c \tau_{1 j}\right) \leqslant e^{\lambda_{11}\left(t-c \tau_{1 j}\right)}$. Noting that for any $k \in \mathbb{Z}_{0}$,

$$
\underline{\Phi}_{1}(\mathrm{t}+\mathrm{k}) \geqslant \mathrm{e}^{\lambda_{11}(\mathrm{t}+\mathrm{k})}-\mathrm{q} \mathrm{e}^{\eta \lambda_{11}(\mathrm{t}+\mathrm{k})},
$$

we have

$$
\begin{aligned}
\underline{L}_{1}(t) \geqslant & \sum_{k \in \mathbb{Z}_{0}} J_{1}(k)\left[e^{\lambda_{11}(t+k)}-e^{\lambda_{11} t}-q e^{\eta \lambda_{11} t}\left(e^{\eta \lambda_{11} k}-1\right)\right]-c\left(e^{\lambda_{11} t}-q e^{\eta \lambda_{11} t}\right)^{\prime} \\
& +r_{1}\left(e^{\lambda_{11} t}-q e^{\eta \lambda_{11} t}\right)\left[1-a_{11}\left(e^{\lambda_{11} t}-q e^{\eta \lambda_{11} t}\right)-\sum_{j \in I_{1}} a_{1 j} e^{\lambda_{11}\left(t-c \tau_{1 j}\right)}\right] \\
= & e^{\lambda_{11} t}\left[\sum_{k \in Z_{0}} J_{1}(k)\left(e^{\lambda_{11} k}-1\right)-c \lambda_{11}+r_{1}\right]-q e^{\eta \lambda_{11} t}\left[\sum_{k \in Z_{0}} J_{1}(k)\left(e^{\eta \lambda_{11} k}-1\right)-c \eta \lambda_{11}+r_{1}\right] \\
& -r_{1}\left(e^{\lambda_{11} t}-q e^{\eta \lambda_{11} t}\right)\left[a_{11}\left(e^{\lambda_{11} t}-q e^{\eta \lambda_{11} t}\right)+\sum_{j \in I_{1}} a_{1 j} e^{\lambda_{11}\left(t-c \tau_{1 j}\right)}\right] \\
\geqslant & -q e^{\eta \lambda_{11} t} \Delta_{1}\left(\eta \lambda_{11}, c\right)-r_{1} a_{11}\left(e^{\lambda_{11} t}-q e^{\eta \lambda_{11} t}\right)^{2}-r_{1} e^{\lambda_{11} t} \sum_{j \in I_{1}} a_{1 j} e^{\lambda_{11}\left(t-c \tau_{1 j}\right)} \\
\geqslant & -q e^{\eta \lambda_{11} t} \Delta_{1}\left(\eta \lambda_{11}, c\right)-r_{1} a_{11} e^{2 \lambda_{11} t}-r_{1} \sum_{j \in I_{1}} a_{1 j} e^{2 \lambda_{11} t} .
\end{aligned}
$$

Noting that $\mathrm{t}_{12}<0$, by (4.8) and (4.13), we have

$$
\underline{\mathrm{L}}_{1}(\mathrm{t}) \geqslant \mathrm{e}^{2 \lambda_{11} \mathrm{t}}\left[-\mathrm{q} \Delta_{1}\left(\eta \lambda_{11}, \mathrm{c}\right)-\mathrm{r}_{1} \mathrm{a}_{11}-\mathrm{r}_{1} \sum_{\mathrm{j} \in \mathrm{I}_{1}} \mathrm{a}_{1 \mathrm{j}}\right] \geqslant 0
$$

When $t>t_{12}$, then $\underline{\phi}_{1}(t)=k_{1}-\left(k_{1}-\frac{\sigma}{2}\right) e^{-\lambda t}, \bar{\phi}_{j}\left(t-c \tau_{1 j}\right) \leqslant k_{j}+k_{j} e^{-\lambda\left(t-c \tau_{1 j}\right)}, j \in I_{1}$. Noting that for 
any $k \in \mathbb{Z}_{0}$,

$$
\underline{\phi}_{1}(t+k) \geqslant k_{1}-\left(k_{1}-\frac{\sigma}{2}\right) e^{-\lambda(t+k)},
$$

we have

$$
\begin{aligned}
\underline{L}_{1}(t) \geqslant & \sum_{k \in \mathbb{Z}_{0}} J_{1}(k)\left[\left(k_{1}-\frac{\sigma}{2}\right) e^{-\lambda t}-\left(k_{1}-\frac{\sigma}{2}\right) e^{-\lambda(t+k)}\right]-c \lambda\left(k_{1}-\frac{\sigma}{2}\right) e^{-\lambda t}+r_{1}\left[k_{1}-\left(k_{1}-\frac{\sigma}{2}\right) e^{-\lambda t}\right] \\
& {\left[1-a_{11}\left[k_{1}-\left(k_{1}-\frac{\sigma}{2}\right) e^{-\lambda t}\right]-\sum_{j \in I_{1}} a_{1 j} k_{j}-\sum_{j \in I_{1}} a_{1 j} k_{j} e^{-\lambda\left(t-c \tau_{1 j}\right)}\right] } \\
\geqslant & e^{-\lambda t}\left\{\left(k_{1}-\frac{\sigma}{2}\right) \sum_{k \in \mathbb{Z}_{0}} J_{1}(k)\left(1-e^{-\lambda k}\right)-c \lambda\left(k_{1}-\frac{\sigma}{2}\right)\right. \\
& \left.+r_{1}\left[k_{1}-\left(k_{1}-\frac{\sigma}{2}\right) e^{-\lambda t}\right]\left[a_{11}\left(k_{1}-\frac{\sigma}{2}\right)-\sum_{j \in I_{1}} a_{1 j} k_{j} e^{\lambda c \tau_{1 j}}\right]\right\} .
\end{aligned}
$$

Due to (4.6) and (4.14), we have

$$
\begin{aligned}
\underline{L}_{1}(t) \geqslant & e^{-\lambda t}\left\{\left(k_{1}-\frac{\sigma}{2}\right) \sum_{k \in \mathbb{Z}_{0}} J_{1}(k)\left(1-e^{-\lambda k}\right)-c \lambda\left(k_{1}-\frac{\sigma}{2}\right)\right. \\
& \left.+\frac{r_{1} \sigma}{4}\left[a_{11}\left(k_{1}-\frac{\sigma}{2}\right)-\sum_{j \in I_{1}} a_{1 j} k_{j} e^{\lambda c \tau_{1 j}}\right]\right\} \\
:= & e^{-\lambda t} I_{2}(\lambda) .
\end{aligned}
$$

By (4.7) and (4.9), we have

$$
\begin{aligned}
I_{2}(0) & =\frac{r_{1} \sigma}{4}\left[a_{11}\left(k_{1}-\frac{m_{1}}{2}\right)-\sum_{j \in I_{1}} a_{1 j} k_{j}\right] \\
& >\frac{r_{1} \sigma}{4}\left(a_{11} \bar{\epsilon}_{1}-\sum_{j \in I_{1}} a_{1 j} k_{j}\right)>0 .
\end{aligned}
$$

Then there exists $\lambda_{2}^{*}>0$ such that $I_{2}(\lambda)>0$ for any $\lambda \in\left(0, \lambda_{2}^{*}\right)$. Thus, $\underline{L}_{1}(t) \geqslant 0$ holds for $\lambda \in\left(0, \lambda_{2}^{*}\right)$ if $t>t_{12}$.

Similarly, we can prove that

$$
\sum_{k \in \mathbb{Z}_{0}} J_{i}(k)\left[\underline{\phi}_{i}(t+k)-\underline{\phi}_{i}(t)\right]-c \underline{\phi}_{i}^{\prime}(t)+r_{i} \underline{\phi}_{i}(t)\left[1-a_{i i} \underline{\phi}_{i}(t)-\sum_{j \in I_{i}} a_{i j} \bar{\phi}_{j}\left(t-c \tau_{i j}\right)\right] \geqslant 0,
$$

for $i=2, \cdots, m$.

By the above argument, we see that $\underline{\phi}_{1}(t), \underline{\phi}_{2}(t), \cdots, \underline{\phi}_{m}(t)$ is a lower solution of (4.3).

By Theorem 3.6, we have the following result.

Theorem 4.8. Assume that (4.2) holds. Then for every c $>\mathrm{c}^{*}$, system (4.1) has a traveling wave solution with speed c connecting the trivial steady state $\mathrm{E}_{0}$ and the positive steady state $\mathrm{E}^{*}$.

\section{Acknowledgment}

This work is supported by Anhui Provincial Natural Science Foundation (No. 1708085QA13), Outstanding Young Talent Support Project of Anhui High School (No. [2014]181) and Natural Science Foundation of Anhui Provincial Education Department (No. KJ2016A517). 


\section{References}

[1] P. W. Bates, A. Chamj, A discrete convolution model for phase transitions, Arch. Ration. Mech. Anal., 150 (1999), 281-305. 1

[2] P. W. Bates, P. C. Fife, X.-F. Ren, X.-F. Wang, Traveling waves in a convolution model for phase transitions, Arch. Rational Mech. Anal., 138 (1997), 105-136. 1

[3] J. Bell, C. Cosner, Threshold behavior and propagation for nonlinear differential-difference systems motivated by modeling myelinated axons, Quart. Appl. Math., 42 (1984), 1-14. 1

[4] J. W. Cahn, S.-N. Chow, E. S. Van Vleck, Spatially discrete nonlinear diffusion equations, Second Geoffrey J. Butler Memorial Conference in Differential Equations and Mathematical Biology, Edmonton, AB, (1992), Rocky Mountain J. Math., 25 (1995), 87-118. 1

[5] J. W. Cahn, J. Mallet-Paret, E. S. Van Vleck, Traveling wave solutions for systems of ODEs on a two-dimensional spatial lattice, SIAM J. Appl. Math., 59 (1998), 455-493. 1

[6] S.-N. Chow, Lattice dynamical systems, Dynamical systems, Lecture Notes in Math., Springer, Berlin, 1822 (2003), 1-102. 1

[7] S.-N. Chow, J. Mallet-Paret, W.-X. Shen, Traveling waves in lattice dynamical systems, J. Differential Equations, 149 (1998), 248-291. 1

[8] J. Fang, J.-J. Wei, X.-Q. Zhao, Spreading speeds and travelling waves for non-monotone time-delayed lattice equations, Proc. R. Soc. Lond. Ser. A Math. Phys. Eng. Sci., 466 (2010), 1919-1934. 1

[9] C.-H. Hsu, S.-S. Lin, Existence and multiplicity of traveling waves in a lattice dynamical system, J. Differential Equations, $164(2000), 431-450.1$

[10] J.-H. Huang, G. Lu, Traveling wave solutions to systems of delayed lattice differential equations,(Chinese) ; translated from Chinese Ann. Math. Ser. A, 25 (2004), 153-164, Chinese J. Contemp. Math., 25 (2004), 125-136. 1

[11] J.-H. Huang, G. Lu, S.-G. Ruan, Traveling wave solutions in delayed lattice differential equations with partial monotonicity, Nonlinear Anal., 60 (2005), 1331-1350. 1

[12] G. Lin, W.-T. Li, Traveling waves in delayed lattice dynamical systems with competition interactions, Nonlinear Anal. Real World Appl., 11 (2010), 3666-3679. 1

[13] G. Lin, W.-T. Li, M.-J. Ma, Traveling wave solutions in delayed reaction diffusion systems with applications to multi-species models, Discrete Contin. Dyn. Syst. Ser. B, 13 (2010), 393-414. 1, 4

[14] G. Lin, W.-T. Li, S.-X. Pan, Travelling wavefronts in delayed lattice dynamical systems with global interaction, J. Difference Equ. Appl., 16 (2010), 1429-1446. 1, 3

[15] Y. Lin, Q.-R. Wang, K. Zhou, Traveling wave solutions in n-dimensional delayed reaction-diffusion systems with mixed monotonicity, J. Comput. Appl. Math., 243 (2013), 16-27. 1

[16] S.-W. Ma, P.-X. Weng, X.-F. Zou, Asymptotic speed of propagation and traveling wavefronts in a non-local delayed lattice differential equation, Nonlinear Anal., 65 (2006), 1858-1890. 1

[17] S.-W. Ma, X.-F. Zou, Propagation and its failure in a lattice delayed differential equation with global interaction, J. Differential Equations, 212 (2005), 129-190. 1

[18] J. Mallet-Paret, Traveling waves in spatially discrete dynamical systems of diffusive type, Dynamical systems, Lecture Notes in Math., Springer, Berlin, 1822 (2003), 231-298. 1

[19] H. F. Weinberger, M. Lewis, B.-T. Li, Analysis of linear determinacy for spread in cooperative models, J. Math. Biol., 45 (2002), 183-218. 1

[20] P.-X. Weng, H.-X. Huang, J.-H. Wu, Asymptotic speed of propagation of wave fronts in a lattice delay differential equation with global interaction, IMA J. Appl. Math., 68 (2003), 409-439. 1

[21] J.-H. Wu, X.-F. Zou, Asymptotic and periodic boundary value problems of mixed FDEs and wave solutions of lattice differential equations, J. Differential Equations, 135 (1997), 315-357. 1

[22] J. Xia, Z.-X. Yu, Y.-C. Dong, H.-Y. Li, Traveling waves for n-species competitive system with nonlocal dispersals and delays, Appl. Math. Comput., 287/288 (2016), 201-213. 1, 4

[23] B. Zinner, Existence of traveling wavefront solutions for the discrete Nagumo equation, J. Differential Equations, 96 (1992), 1-27. 1

[24] B. Zinner, G. Harris, W. Hudson, Traveling wavefronts for the discrete Fisher's equation, J. Differential Equations, 105 (1993), 46-62. 1 